\title{
Erratum: Improved real-space genetic algorithm for crystal structure and polymorph prediction [Phys. Rev. B 77, 134117 (2008)]
}

\author{
N. L. Abraham and M. I. J. Probert \\ (Received 20 July 2016; published 22 August 2016)
}

DOI: 10.1103/PhysRevB.94.059904

In our earlier work, there was an error in the derivation of the spherically averaged scattering intensity, presented as Eq. (5) in the original paper. This equation should have read

$$
\Lambda\left(k_{r}\right)=\sum_{n=1}^{N} \rho^{\prime}(n)^{2}+2 \sum_{n=1}^{N} \sum_{m>n}^{N} \rho^{\prime}(n) \rho^{\prime}(m) j_{0}\left(k_{r}\left|\mathbf{r}_{n}-\mathbf{r}_{m}\right|\right),
$$

where $j_{0}(r)$ is the spherical Bessel function of the first kind.

The consequence of using the incorrect equation is that the $R$ factor behaved significantly differently for calculations with varying volumes and had an imbalance between the two terms which skewed the results.

Figure 1 shows the $R$ factor when comparing a reference eight-atom silicon unit cell to a perturbed copy of that cell. In the replica, the atomic positions may be randomly perturbed up to some amount $\Delta x$ with the cell vectors fixed, or both atomic positions and cell vectors may be randomly perturbed up to some amount $\Delta x$. The original equation can give significantly different results for similar structures in fixed and variable cell calculations.

As a result of this error, structures of different volumes will give incorrect $R$ factors. This may have slowed the convergence of the genetic algorithm calculations presented in the original paper but will not have changed the final predicted structures.

The error was detected, and the correct equation derived by E. J. Higgins, University of York, U.K.

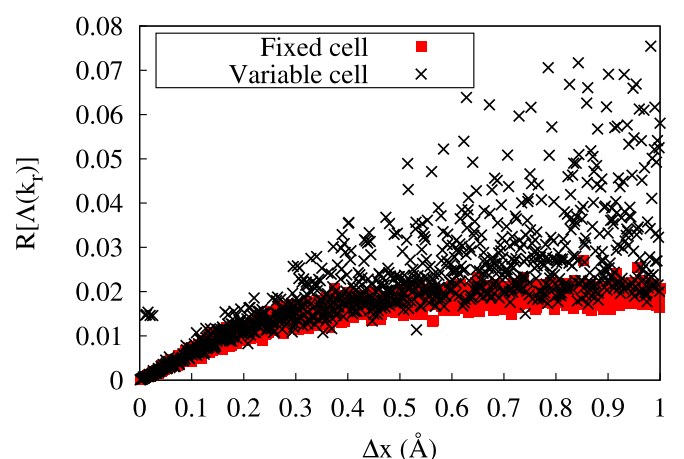

(a)

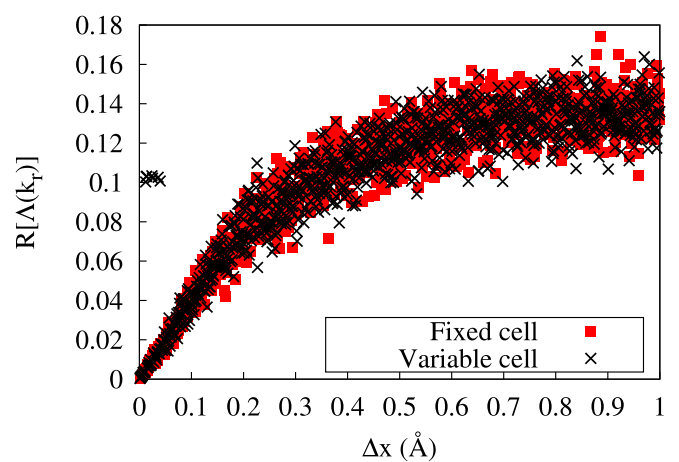

(b)

FIG. 1. $R$ factor vs perturbation size for an eight-atom unit cell using (a) the original and (b) the corrected scattering intensities. 